\title{
Dileptons from hot heavy static photons
}

\author{
P. Aurenche* \\ Laboratoire d'Annecy-le-Vieux de Physique Théorique, 74941 Annecy-le-Vieux, France \\ M. E. Carrington ${ }^{\dagger}$ \\ Department of Physics, Brandon University, Manitoba, R7A 6A9 Canada \\ N. Marchal \\ Laboratoire d'Annecy-le-Vieux de Physique Théorique, 74941 Annecy-le-Vieux, France
}

October 30, 2018

\begin{abstract}
We compute the production rate of lepton pair by static photons at finite temperature at two-loop order. We treat the infrared region of the gluon phase space carefully by using a hard thermal loop gluon propagator. The result is free of infrared and collinear divergences and exhibits an enhancement which produces a result of order $\sim e^{2} g^{3}$ instead of $\sim e^{2} g^{4}$ as would be expected from ordinary perturbation theory.
\end{abstract}

\section{Introduction}

The production of photons and leptons at finite temperature is studied as a signal for the formation of a quark-gluon plasma in heavy ion collisions [1, 2, 3, 4, 5, 6, 7, 7. Photons and leptons are expected to escape easily from nuclear matter because of their large mean free path. Computations are done in two steps. The first step is to compute the production rate at a given order in perturbative thermal field theory. The second step is to substitute this result into a hydrodynamical model that simulates the experimentally realized heavy ion collision 4, 5, 6, 7]. In this paper we discuss only the first of these steps.

The production rate of lepton pairs by a photon of virtuality $Q^{2}$ is 8

$$
\frac{d N}{d q_{0} d^{3} q d^{4} x}=\frac{\alpha}{12 \pi^{3}} \frac{1}{Q^{2}} n_{B}\left(q_{0}\right) \operatorname{Im} \Pi_{R}\left(q_{0}, q\right)^{\mu}{ }_{\mu}
$$

Note that there are two coupling constants involved in this equation, or equivalently, two fine structure constants: $e$ or $\alpha$ characterizes the electromagnetic interaction, and $g$ or $\alpha_{s}$ characterizes the strong interaction. The formula above is valid to first order in $\alpha$ and arbitrary order in $\alpha_{s}$, although the actual calculation will involve a perturbative expansion of the polarization tensor.

In this paper we are interested in contributions to lepton pair production by heavy static photons. We take the photon momentum to be: $Q^{\mu}=\left(q_{0}, \overrightarrow{0}\right)$ with $Q^{2}=q_{0}^{2} \gg g^{2} T^{2}$. At the Born level, the imaginary part of the trace of the polarization tensor is given by :

$$
\operatorname{Im} \Pi_{R}\left(q_{0}, \overrightarrow{0}\right)_{\mu}^{\mu}=\alpha N_{c} q_{0}^{2}\left[1-2 n_{F}\left(\frac{q_{0}}{2}\right)\right],
$$

which we denote, for simplicity, $\operatorname{Im} \Pi^{\text {Born }} . N_{c}$ is the number of colors.

The rate of static lepton pair production has also been calculated at the two-loop level in the bare theory. In physical terms these corrections include the contributions of Compton scattering, quark-antiquark

\footnotetext{
${ }^{*}$ Electronic address: aurenche@lapp.in2p3.fr

${ }^{\dagger}$ Electronic address: carrington@brandonu.ca
} 
annihilation into a virtual photon, and the $3 \rightarrow 1$ process $q \bar{q} G \rightarrow \gamma^{*}$. In the large $q_{0}^{2} / T^{2}$ limit, the result has been found to be [9]:

$$
\operatorname{Im} \Pi_{R}\left(q_{0}, \overrightarrow{0}\right)^{\mu}{ }_{\mu}=\operatorname{Im} \Pi^{\text {Born }} \sqrt{1-\frac{4 m_{\mathrm{q}}^{2}}{q_{0}^{2}}}\left[1+\frac{2 m_{\mathrm{q}}^{2}}{q_{0}^{2}}+C_{F} \frac{3}{4} \frac{g^{2}}{4 \pi^{2}}\right]
$$

where $m_{\mathrm{q}}^{2}=C_{F} g^{2} T^{2} / 4$ is the thermal quark mass generated by the one-loop self-energy corrections, with $C_{F}=4 / 3$ the usual QCD structure constant. The temperature independent term is simply the $T=0$ first order QCD correction to the process $e^{+} e^{-} \rightarrow q \bar{q}$ with massless kinematics. The square root threshold factor in eq. (1.3) is obtained if the thermal self-energy corrections are resummed on the quark lines. The interpretation of the temperature dependent corrections is very simple. Starting from massless quarks the thermal corrections, in the considered limit, are obtained by simply considering the production in the vacuum of quarks with a thermal mass. This result supports the quasi-particle interpretation of thermal effects. In the strict perturbative sense, which we adhere to here, the threshold factor can be expanded in $m_{\mathrm{q}}^{2} / q_{0}^{2}$ and one observes a cancellation of thermal corrections at $\mathcal{O}\left(g^{2}\right)$ : the first thermal corrections are then expected to appear $\mathcal{O}\left(g^{4}\right)$,

$$
\operatorname{Im} \Pi_{R}\left(q_{0}, \overrightarrow{0}\right)^{\mu}{ }_{\mu}=\operatorname{Im} \Pi^{\text {Born }}\left[1+C_{F} \frac{3}{4} \frac{g^{2}}{4 \pi^{2}}+\mathcal{O}\left(g^{4} \frac{T^{4}}{q_{0}^{4}}\right)\right]
$$

These calculations are done by integrating over the full fermion and gluon phase space including contributions from both hard and soft modes. However, it is well known that it is inconsistent to use bare propagators at soft momentum scales $(p \sim g T)$. For soft momenta, collective modes play an important role and hard thermal loop (HTL) resummed propagators must be used 10, 11, 12. We expect that this problem is more serious for gluon modes than for fermion modes. Gluons are bosons and carry a thermal factor of the Bose-Einstein form which has the limiting form $T / l_{0} \sim 1 / g \gg 1$ when $l_{0}$ (the gluon energy) is $\sim g T$. In contrast, fermions carry a thermal factor of the Fermi-Dirac form which approachs $1 / 2$ in the high temperature limit. Thus we expect that collective effects due to soft gluons give rise to an enhancement.

In this article we will calculate the two loop photon polarization tensor using a HTL gluon propagator in order to study the importance of the soft part of the gluon phase space (Fig. [1). When the resummed gluon propagator is used the physics is changed in the following ways:

[1] transverse and longitudinal modes have different behaviour and must be considered separately;

[2] in the time-like region gluons become massive quasi-particles;

[3] in the space-like region the gluon polarization tensor has a non-zero imaginary part which leads to Landau damping. This damping is associated with new physical processes which do not appear at the bare two loop level and correspond to the scattering of quarks and gluons in the medium.

One interesting aspect of the study of photon and lepton pair production at finite temperature is the treatment of infrared and collinear divergences. At zero temperature there is a general result (the KLN theorem 13, 14]) which insures the cancellation of these divergences. At finite temperature, the problem is complicated by the presence of the collective modes. In all calculations divergences are removed through cancellations that are similar to those that occur at zero temperature, however, there is no generalized version of the KLN theorem that applies at finite temperature.

In bare perturbation theory, it has been shown that the corresponding diagrams are finite with respect to collinear and infrared divergences [15, 16, 17, 18, 19. The case of a non-static photon has been studied in [20, 21]. A calculation using resummed propagators has been done in 22. The authors use a scalar external particle, bare fermion propagators and a HTL gluon propagator. They find that all divergences cancel, and that the contribution from longitudinal gluon modes is enhanced relative to the contribution from transverse gluon modes by one power of the coupling constant. The transverse mode gives a contribution to the polarization tensor of order $e^{2} \mathcal{O}\left(g^{4}\right)$, as is expected from perturbation theory, and the longitudinal mode gives a contribution of order $e^{2} \mathcal{O}\left(g^{3}\right)$. In this paper, we perform the corresponding calculation for a virtual photon (for which the result is related to the dilepton production rate (1.1) which is a physical quantity). We find that the cancellation of divergences has the same structure as in the scalar theory, but we obtain an enhanced result $\sim e^{2} g^{3}$ in both the longitudinal and transverse sectors.

This paper is organised as follows : in Sec. 2 we review some elements of real time finite temperature field theory and of the hard thermal loop effective theory. In Sec. 3 we outline the calculation of the 2-loop 
diagrams. We discuss some preliminary results in Sec. 4. In Sec. [5 we perform the final integrals over the gluon energy and momentum. Finally, in Sec. 6] we present some conclusions.

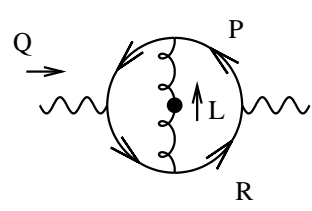

(a)

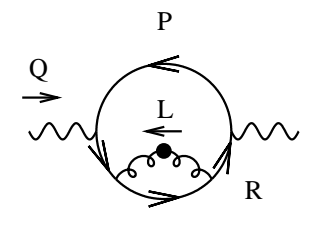

(b)

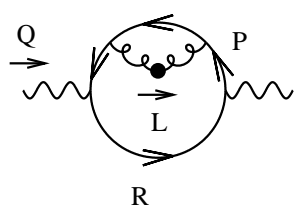

(c)

Figure 1: Vertex (a) and self-energy ((b) and (c)) diagrams

\section{Hard thermal loops}

We consider the case of hard fermions and soft gluons. Fermion propagators have momenta of order $p \gg g T$, and are not modified by collective effects. The fermionic propagator is given by

$$
S(P)=\not P D(P) ; \quad D(P)=\frac{i}{P^{2}}=\frac{i}{p_{0}^{2}-\vec{p}^{2}}
$$

Retarded and advanced functions are obtained by taking the appropriate $i \epsilon$ prescription

$$
D(P) \rightarrow D_{R / A}(P)=\frac{i}{\left(p_{0} \pm i \epsilon\right)^{2}-\vec{p}^{2}}
$$

We also define the spectral function and the principal part,

$$
\begin{aligned}
& \rho(P)=D_{R}(P)-D_{A}(P)=2 \pi \epsilon\left(p_{0}\right) \delta\left(P^{2}\right)=-\frac{\pi}{p} \sum_{n= \pm 1} n \delta\left(p_{0}+n p\right) \\
& \mathcal{P}(P)=\frac{1}{2}\left(\frac{1}{\left(p_{0}+i \epsilon\right)^{2}-p^{2}}+\frac{1}{\left(p_{0}-i \epsilon\right)^{2}-p^{2}}\right)
\end{aligned}
$$

which gives,

$$
\begin{aligned}
& D_{R}(P)=i \mathcal{P}(P)+\frac{1}{2} \rho(P) \\
& D_{A}(P)=i \mathcal{P}(P)-\frac{1}{2} \rho(P)
\end{aligned}
$$

The exchanged gluon is soft, and thus the propagator will be modified by collective effects. We use a hard thermal loop effective gluon propagator. The gluon propagator is diagonal in color, and color indices will be omitted. We work in the strict Coulomb gauge and decompose the gluon propagator into transverse and longitudinal pieces:

$$
-D_{R / A}^{\mu \nu}(L)=P_{T}^{\mu \nu}(L) \Delta_{R / A}^{T}(L)+P_{L}^{\mu \nu}(L) \Delta_{R / A}^{L}(L)
$$

The projectors are defined as 23] :

$$
\begin{aligned}
& P_{T}^{\mu \nu}(L)=\left(\begin{array}{cc}
0 & 0 \\
0 & -\delta^{i j}+\hat{l}^{i} \hat{l}^{j}
\end{array}\right) \\
& P_{L}^{\mu \nu}(L)=-\frac{L^{2}}{l^{2}}\left(\begin{array}{ll}
1 & 0 \\
0 & 0
\end{array}\right)
\end{aligned}
$$


and coefficients are:

$$
\Delta^{T / L}(L)=\frac{i}{L^{2}-\Pi_{T / L}(L)}
$$

The quantities $\Pi_{T / L}(L)$ are defined by choosing the decomposition of the gluon polarization tensor that satisfies the usual Schwinger-Dyson equation:

$$
\Pi^{\mu \nu}(L)=P_{T}^{\mu \nu}(L) \Pi_{T}(L)+P_{T}^{\mu \nu}(L) \Pi_{T}(L)=i\left(\left(D^{\mu \nu}(L)\right)^{-1}-\left(D_{0}^{\mu \nu}(L)\right)^{-1}\right)
$$

where $D_{0}^{\mu \nu}(L)$ is obtained from (2.3) by setting the polarization tensor to zero. We note that (2.6) holds separately for retarded and advanced components. The subscripts $R / A$ have been omitted to simplify the notation. We use the 1-loop HTL results [24, 25] :

$$
\begin{aligned}
& \Pi_{T}(l x, l)=3 m_{g}^{2}\left[\frac{x^{2}}{2}+\frac{x\left(1-x^{2}\right)}{4} \ln \left(\frac{x+1}{x-1}\right)\right] \\
& \Pi_{L}(l x, l)=3 m_{g}^{2}\left(1-x^{2}\right)\left[1-\frac{x}{2} \ln \left(\frac{x+1}{x-1}\right)\right]
\end{aligned}
$$

where $x=l_{0} / l$ and $m_{g}^{2}=g^{2} T^{2}\left[N+N_{f} / 2\right] / 9$ is the soft gluon thermal mass in a $S U(N)$ gauge theory with $N_{f}$ flavors. The spectral function is defined as

$$
\rho_{T / L}\left(l_{0}, l\right)=\Delta_{R}^{T / L}(L)-\Delta_{A}^{T / L}(L) .
$$

\section{Calculation}

We want to calculate the trace of the imaginary part of the retarded photon polarization tensor which is given by

$$
\operatorname{Im}\left(\Pi_{R}\right)_{\mu}^{\mu}=\frac{1}{2 i}\left(\left(\Pi_{R}\right)_{\mu}^{\mu}-\left(\Pi_{A}\right)_{\mu}^{\mu}\right)
$$

The diagrams to be evaluated are shown in Fig. [1]. Since we consider the production of a static external photon $\left(Q^{\mu}=\left(q_{0}, \overrightarrow{0}\right)\right)$, with $q_{0}>0$, all the azimuthal angular integrations can be carried out trivially and the integration measure is rewritten as,

$$
\int d^{4} P \int d^{4} L=8 \pi^{2} \int d l_{0} \int d l l^{2} \int d y \int d p_{0} \int d p p^{2}
$$

where $y=\hat{l} \cdot \hat{p}$ is related to the angle between the gluon and quark momenta. We simplify the notation by defining the momentum variable $R=P+Q$.

\subsection{Vertex diagram}

The vertex contribution (Fig. [1a]) to the imaginary part of the photon polarization tensor can be written as 26, 27, 28:

$$
\begin{aligned}
\operatorname{Im}_{R}^{(\text {vertex })_{\mu}^{\mu}=-N_{c} C_{F} \frac{e^{2} g^{2}}{2}} \int \frac{d^{4} P}{(2 \pi)^{4}}\left[n_{F}\left(r_{0}\right)-n_{F}\left(p_{0}\right)\right] \int \frac{d^{4} L}{(2 \pi)^{4}} \operatorname{Tr}_{\rho \sigma}^{(\text {vertex })} n_{B}\left(l_{0}\right) \mathcal{R}^{\rho \sigma} \\
\times\left[D_{R}(P) D_{R}(P+L)-D_{A}(P) D_{A}(P+L)\right] \\
\times\left[D_{R}(R) D_{R}(R+L)-D_{A}(R) D_{A}(R+L)\right]
\end{aligned}
$$

where we have kept only terms proportional to the gluon thermal factor $n_{B}\left(l_{0}\right)$ since we are interested in a possible enhancement from the soft part of the gluon phase space. Rewriting this expression using (2.2) we 
obtain,

$$
\begin{aligned}
& \operatorname{Im} \Pi_{R}^{(\text {vertex })_{\mu}^{\mu}}=N_{c} C_{F} e^{2} g^{2} \int \frac{d^{4} P}{(2 \pi)^{4}}\left[n_{F}\left(r_{0}\right)-n_{F}\left(p_{0}\right)\right] \rho(P) \int \frac{d^{4} L}{(2 \pi)^{4}} \operatorname{Tr}_{\rho \sigma}^{(v e r t e x)} n_{B}\left(l_{0}\right) \mathcal{R}^{\rho \sigma} \\
& \times \mathcal{P}(L+P)[\rho(R+L) \mathcal{P}(R)+\rho(R) \mathcal{P}(R+L)]
\end{aligned}
$$

where we have defined

$$
\mathcal{R}^{\rho \sigma}=\rho_{T}(L) P_{T}^{\rho \sigma}(L)+\rho_{L}(L) P_{L}^{\rho \sigma}(L),
$$

and the Dirac trace is calculated to be:

$$
\begin{aligned}
\operatorname{Tr}_{\rho \sigma}^{(\text {vertex })}=\quad & \operatorname{Tr}\left[\not P \gamma^{\mu} R \gamma_{\rho}(R+\not L) \gamma_{\mu}(\not P+L) \gamma_{\sigma}\right] \\
=\quad & -8\left(2 L \cdot P L \cdot R+L \cdot R P^{2}+L \cdot P R^{2}+P^{2} R^{2}-L^{2} R \cdot P\right) g_{\rho \sigma} \\
& +16\left(R^{2}\left(L_{\rho}+P_{\rho}\right) P_{\sigma}+P^{2}\left(L_{\rho}+R_{\rho}\right) R_{\sigma}+L \cdot R P_{\rho}\left(L_{\sigma}+P_{\sigma}-R_{\sigma}\right)\right. \\
& +L \cdot P R_{\rho}\left(L_{\sigma}+R_{\sigma}-P_{\sigma}\right)-L^{2} P_{\rho} R_{\sigma}-R \cdot P\left(P_{\rho} R_{\sigma}+\left(L_{\rho}+P_{\rho}\right)\left(L_{\sigma}+R_{\sigma}\right)\right) .
\end{aligned}
$$

By analogy with the zero temperature calculation, the first term in eq. (3.3) is called the real cut (the fermion propagators $P$ and $R+L$ are cut) while the second term is called the virtual cut ( $P$ and $R$ are cut). There are two additional cuts: one where the fermion propagators carrying momenta $P+L$ are $R$ are cut, and one where the propagators carrying momenta $P+L$ and $R+L$ are cut. Using symmetry arguments one can show that these two terms contribute an overall factor of two which has been included in (3.3). The contraction $P_{T / L}^{\rho \sigma} \operatorname{Tr}_{\rho \sigma}^{(\text {vertex })}$ is straightforward to perform. We look at the longitudinal and transverse parts separately. We obtain,

$$
\begin{aligned}
P_{L}^{\rho \sigma} \operatorname{Tr}_{\rho \sigma}^{(v e r t e x)}= & 8\left(1-x^{2}\right)\left[q_{0}^{2}\left(2 p^{2}+l\left(p_{0} x+p y\right)-q_{0} l\left(-2 p^{2} x+p_{0}\left(l\left(1-x^{2}\right)+2 p y\right)\right)-2 l^{2} p^{2}\left(1-y^{2}\right)\right]\right. \\
P_{T}^{\rho \sigma} \operatorname{Tr}_{\rho \sigma}^{(v e r t e x)}= & 16\left[q_{0}^{2}\left(p^{2}\left(1-y^{2}\right)+l\left(p_{0} x-p y\right)\right)+q_{0}\left(l^{2}\left(p_{0}\left(1+x^{2}\right)-2 p x y\right)-2 l p\left(p_{0} y-p x\right)\right)\right. \\
& \left.-l^{2} p\left(4 p_{0} x y-p\left(1+x^{2}\right)\left(1+y^{2}\right)\right)\right]
\end{aligned}
$$

where we have used $x:=l_{0} / l$ and set $P^{2}=0$ in anticipation of the fact that the factor $\rho(P)$ in (3.3) will give $P^{2}=0$ when (2.1) is used.

\section{$3.2 \quad$ Self-energy graph}

The expression for the self-energy diagrams is similar. We obtain the following expression for the diagram of Fig. [1]:

$$
\begin{aligned}
\operatorname{Im} \Pi_{R}^{\text {self(b) }{ }_{\mu}^{\mu}=-N_{c} C_{F}} \frac{e^{2} g^{2}}{2} \int \frac{d^{4} P}{(2 \pi)^{4}}\left[n_{F}\left(r_{0}\right)-n_{F}\left(p_{0}\right)\right] \int \frac{d^{4} L}{(2 \pi)^{4}} \operatorname{Tr}_{\rho \sigma}^{(\text {self(b)) }} n_{B}\left(l_{0}\right) \mathcal{R}^{\rho \sigma} \\
\times\left[D_{R}(P)-D_{A}(P)\right]\left[\left(D_{R}(R)\right)^{2} D_{R}(R+L)-\left(D_{A}(R)\right)^{2} D_{A}(R+L)\right]
\end{aligned}
$$

where, as before, we have kept only the terms proportional to $n_{B}\left(l_{0}\right)$. Rewriting using (2.2) we obtain,

$$
\begin{aligned}
& \operatorname{Im} \Pi_{R}^{\text {self }(b)^{\mu}}{ }_{\mu}=N_{c} C_{F} e^{2} g^{2} \int \frac{d^{4} P}{(2 \pi)^{4}}\left[n_{F}\left(r_{0}\right)-n_{F}\left(p_{0}\right)\right] \rho(P) \int \frac{d^{4} L}{(2 \pi)^{4}} \operatorname{Tr}_{\rho \sigma}^{(s e l f(b))} n_{B}\left(l_{0}\right) \mathcal{R}^{\rho \sigma} \\
& \times\left[\mathcal{P}(R+L) \mathcal{P}(R) \rho(R)+(\mathcal{P}(R))^{2} \frac{1}{2} \rho(R+L)\right]
\end{aligned}
$$

As in the case of the vertex diagram, the first term in the square bracket corresponds to the real cut, and the second term corresponds to the virtual one. The Dirac trace is:

$$
\begin{aligned}
\operatorname{Tr}_{\rho \sigma}^{\text {self }(b)} & =\operatorname{Tr}\left[\not P \gamma^{\mu} R \gamma_{\rho}(R+\not L) \gamma_{\sigma} R \gamma_{\mu}\right] \\
& =8\left(g_{\rho \sigma}\left(R^{2}(R \cdot P-L \cdot P)+2 L \cdot R R \cdot P\right)+2\left(L_{\rho}+R_{\rho}\right)\left(R^{2} P_{\sigma}-2 R \cdot P R_{\sigma}\right)\right)
\end{aligned}
$$


The expression for the second self-energy diagram (Fig. $\llbracket c]$ ) can be obtained in the same way and it is taken into account by multiplying eq. (3.7) by a factor two. Contracting $P_{T / L}^{\rho \sigma} \operatorname{Tr}_{\rho \sigma}^{\text {self(b) }}$ we obtain,

$$
\begin{aligned}
& P_{L}^{\rho \sigma} \operatorname{Tr}_{\rho \sigma}^{\text {self(b) }}=8\left(1-x^{2}\right) q_{0}^{2}\left(q_{0} p_{0}+l\left(p_{0} x-p y\right)\right) \\
& P_{T}^{\rho \sigma} \operatorname{Tr}_{\rho \sigma}^{\text {self(b) }}=-16 q_{0}^{2}\left(p_{0} q_{0}+\left(l\left(p_{0} x+p y\right)+p^{2}\left(1+y^{2}\right)\right)\right.
\end{aligned}
$$

where we have set $P^{2}=0$ as before.

The first step in the calculation of (3.3) and (3.7) is to perform the $p_{0}$ integration using (2.1). The choice $q_{0}>0$ means that only $n=1$ is kinematically allowed. The second step is to use the delta functions contained in the spectral functions $\rho(R+L)$ and $\rho(R)$ to perform the $p$ integrations in the real and virtual terms respectively. The structure of the real cut is the same for both vertex and self energy diagrams. Making use of (2.1) we have,

$$
\rho(R+L)=\frac{\pi}{l_{0}+q_{0}+l y} \delta\left(p-p_{\text {real }}\right) ; \quad p_{\text {real }}=\frac{\left(l_{0}+q_{0}\right)^{2}-l^{2}}{2\left(l_{0}+q_{0}+l y\right)}
$$

Similarly, we attack the virtual cut by using (2.1) to obtain (remember that $r_{0}=q_{0}+p_{0}$ and $r=p$ in static photon kinematics),

$$
\rho(R)=\frac{\pi}{q_{0}} \delta\left(p-p_{\text {virtual }}\right) ; \quad p_{\text {virtual }}=\frac{q_{0}}{2}
$$

This expression can be used directly to do the $p$ integral in the virtual part of the vertex diagram. The structure of the virtual part of the self energy diagram is more complicated: there is a singularity because of the presence of the factor $\mathcal{P}(R) \rho(R)$. This singularity can be regulated in the standard way [29]. We write

$$
\left(\frac{1}{x+i \epsilon}\right)^{2}=-\frac{d}{d x}\left(\frac{1}{x+i \epsilon}\right) \text {. }
$$

Taking the imaginary part of this equation gives,

$$
\mathcal{P}\left(\frac{1}{x}\right) \delta(x)=-\frac{1}{2} \frac{d}{d x} \delta(x)
$$

Using this expression we obtain,

$$
\mathcal{P}(R) \rho(R)=\frac{\pi}{4 q_{0}^{2}} \frac{d}{d p} \delta\left(p-p_{\text {virtual }}\right)
$$

and perform an integration by parts to remove the derivative from the delta function.

Once the variables $p_{0}$ and $p$ have been integrated over, we can simplify the result by performing an expansion in $l_{0} / q_{0}$ and $l / q_{0}$. However, as shown below, one finds that the leading order term cancels between the real and virtual contributions to each diagram, and thus we must expand to next-to-leading order. The fact that $p_{\text {real }}$ is a function of $l_{0}, l$ and $q_{0}$ means that the contributions from the real cuts will involve an expansion of the $\left(n_{F}\left(r_{0}\right)-n_{F}\left(p_{0}\right)\right)$ thermal factors. We use the notation:

$$
n_{F}=\frac{1}{e^{\beta q_{0} / 2}+1} ; \quad n_{F}^{\prime}=0 ; \quad n_{F}^{\prime \prime}=n_{F}\left(1-n_{F}\right)\left(1-2 n_{F}\right)
$$

After the expansion has been performed, the resulting expression can be integrated over $y$ by hand. We look at longitudinal and transverse pieces separately.

\section{Preliminary Results}

We list below the results for the trace of the imaginary part of the retarded polarization tensor. To simplify the notation, a common factor has been extracted from each term:

$$
\mathrm{FAC}=-\frac{e^{2} g^{2}}{16 \pi^{4}} N_{c} C_{F} \int d l l \int d x n_{B}(x l) \rho_{L / T}(l x, l)
$$


In addition, we have used the notation:

$$
\mathrm{LOG}=\ln \left|\frac{x+1}{x-1}\right| ; \quad x=\frac{l_{0}}{l}
$$

We obtain:

$$
\begin{aligned}
\operatorname{Vertex~}_{L}^{\text {virtual }}= & \left(1-2 n_{F}\right) \frac{1-x^{2}}{x}\left(q_{0}^{2} \mathrm{LOG}-2 l^{2}\left(2 x+\left(1-x^{2}\right) \mathrm{LOG}\right)\right. \\
\operatorname{Vertex}_{L}^{\text {real }}= & -\left(1-2 n_{F}\right) \frac{1-x^{2}}{x}\left[q_{0}^{2} \mathrm{LOG}-2 l^{2}\left(x+\left(1-x^{2}\right) \mathrm{LOG}\right]\right. \\
& \quad-\frac{1}{2} l^{2} \frac{q_{0}^{2}}{T^{2}}\left(1-x^{2}\right)(1-x \mathrm{LOG}) n_{F}^{\prime \prime} \\
\operatorname{Vertex}_{L}= & -l^{2}\left(1-x^{2}\right)\left(2\left(1-2 n_{F}\right)+\frac{1}{2} \frac{q_{0}^{2}}{T^{2}}(1-x \mathrm{LOG}) n_{F}^{\prime \prime}\right)
\end{aligned}
$$

$$
\begin{aligned}
\text { Self }- \text { Energy }_{L}^{\text {virtual }}= & 2\left(1-2 n_{F}\right) q_{0}\left(q_{0}+2 l x\right) \\
\text { Self }- \text { Energy }_{L}^{\text {real }}= & -2\left(1-2 n_{F}\right)\left[q_{0}^{2}+2 l q_{0} x-l^{2}\left(1-x^{2}\right)\right]-\frac{1}{2} l^{2} \frac{q_{0}^{2}}{T^{2}}\left(1-x^{2}\right)\left(1+\frac{2 x l}{q_{0}}\right) n_{F}^{\prime \prime} \\
\text { Self }- \text { Energy }_{L}= & l^{2}\left(1-x^{2}\right)\left(2\left(1-2 n_{F}\right)-\frac{1}{2} \frac{q_{0}^{2}}{T^{2}}\left(1+\frac{2 x l}{q_{0}}\right) n_{F}^{\prime \prime}\right) \\
\text { LONG }= & -\frac{1}{2} l^{2} \frac{q_{0}^{2}}{T^{2}}\left(1-x^{2}\right)\left(2-x \mathrm{LOG}+\frac{2 l x}{q_{0}}\right) n_{F}^{\prime \prime} \\
\text { Vertex }_{T}^{\text {virtual }}= & \left(1-2 n_{F}\right) \frac{1}{x}\left(q_{0}^{2}-2 l^{2}\right)\left(2 x+\left(1-x^{2}\right) \mathrm{LOG}\right) \\
\text { Vertex }_{T}^{\text {real }}= & -\left(1-2 n_{F}\right) \frac{1}{x}\left[q_{0}^{2}\left(2 x+\left(1-x^{2}\right) \mathrm{LOG}\right)-2 l^{2}\left(1-x^{2}\right) \mathrm{LOG}\right] \\
\text { Vertex }_{T} & -l^{2}\left(4\left(1-2 n_{F}\right)+\frac{q_{0}^{2}}{T^{2}}\left(\frac{1}{3}\left(1-3 x^{2}\right)-\frac{1}{2} x\left(1-x^{2}\right) \mathrm{LOG}\right) n_{F}^{\prime \prime}\right)
\end{aligned}
$$

$$
\begin{aligned}
\text { Self }- \text { Energy }_{T}^{\text {virtual }} & =-2\left(1-2 n_{F}\right)\left[q_{0}^{2}(2-x \mathrm{LOG})+l^{2} x\left(2 x-\left(1+x^{2}\right) \mathrm{LOG}\right]\right. \\
\text { Self }- \text { Energy }_{T}^{\text {real }} & =2\left(1-2 n_{F}\right)\left[(2-x \mathrm{LOG})\left(q_{0}^{2}+l^{2}\left(1+x^{2}\right)\right)\right]+\frac{1}{3} \frac{q_{0}^{2}}{T^{2}} l^{2} n_{F}^{\prime \prime} \\
\text { Self }- \text { Energy }_{T} & =l^{2}\left(4\left(1-2 n_{F}\right)+\frac{1}{3} \frac{q_{0}^{2}}{T^{2}} n_{F}^{\prime \prime}\right)
\end{aligned}
$$$$
\text { TRANS }=\frac{1}{2} l^{2} \frac{q_{0}^{2}}{T^{2}} x\left(2 x+\left(1-x^{2}\right) \mathrm{LOG}\right) n_{F}^{\prime \prime}
$$ 
Before proceeding, we make the following observations about these results:

[1]: In each of the four cases: (vertex/self-energy) $\times$ (longitudinal/transverse), the leading order term in the $l / q_{0}$ expansion cancels between real and virtual contributions and the final result is of order $l^{2}$.

[2]: For both the longitudinal and transverse pieces the term proportional to $\left(1-2 n_{F}\right)$ cancels between the self energy and vertex contributions.

[3]: The longitudinal propagator has the form $\Delta_{L}(L) \sim 1 /\left(1-x^{2}\right)$, as can be seen from (2.5) and (2.7). Consequently, the terms (4.5) and (4.6) exhibit co-linear singularities in the form of a factor $1 /\left(1-x^{2}\right)$ which diverges when $x \rightarrow \pm 1$. When the two expressions are combined however, the sum (4.7) is well behaved.

As a check on these results, we sketch in the appendix an alternative derivation of the transverse gluon contribution.

\section{$5 \quad l_{0}$ and $l$ integrals}

In this section we perform the two remaining integrals over $l_{0}$ and $l$. From (4.1), (4.8) and (4.15) and using the approximation $n_{B}(l x) \sim T /(l x)$ we have,

$$
\begin{aligned}
& L O N G=-\frac{e^{2} g^{2}}{(2 \pi)^{4}} N_{c} C_{F} \frac{q_{0}^{2}}{T} n_{F}^{\prime \prime} I_{L} \\
& \text { TRANS }=-\frac{e^{2} g^{2}}{(2 \pi)^{4}} N_{c} C_{F} \frac{q_{0}^{2}}{T} n_{F}^{\prime \prime} I_{T}
\end{aligned}
$$

where,

$$
\begin{aligned}
& I_{L}=\int_{-\infty}^{\infty} \frac{d x}{x} \int_{0}^{\infty} d l l^{2} \rho_{L}(l x, l)\left[\left(1-x^{2}\right) \frac{x}{2} \ln \left|\frac{x+1}{x-1}\right|-1\right] \\
& I_{T}=\int_{-\infty}^{\infty} \frac{d x}{x} \int_{0}^{\infty} d l l^{2} \rho_{T}(l x, l)\left[x^{2}+\left(1-x^{2}\right) \frac{x}{2} \ln \left|\frac{x+1}{x-1}\right|\right]
\end{aligned}
$$

It is interesting to remark that the terms in square brackets in the integrands are proportional to $\operatorname{Re} \Pi_{L}$ and Re $\Pi_{T}$ respectively.

\subsection{Longitudinal Integral}

We consider first the longitudinal integral (5.1). We divide the integral into two pieces, one with and one without the logarithm:

$$
\begin{aligned}
& I_{L}^{a}=-\int_{-\infty}^{\infty} \frac{d x}{x} \int_{0}^{\infty} d l l^{2} \rho_{L}(l x, l)\left(1-x^{2}\right) \\
& I_{L}^{b}=\int_{-\infty}^{\infty} \frac{d x}{x} \int_{0}^{\infty} d l l^{2} \rho_{L}(l x, l)\left(1-x^{2}\right) \frac{x}{2} \ln \left|\frac{x+1}{x-1}\right|
\end{aligned}
$$

The calculation of the first piece is standard 28]. One obtains

$$
I_{L}^{a}=\sqrt{3} \pi^{2} m_{g}
$$

To calculate $I_{L}^{b}$ we use the method proposed in 22]. We use an integral representation for the logarithm and write,

$$
\ln \left|\frac{x+1}{x-1}\right|=\int_{-1}^{1} d x^{\prime} \mathcal{P}\left(\frac{1}{x-x^{\prime}}\right)
$$

where the principal part is defined as

$$
\mathcal{P}\left(\frac{1}{x}\right)=\frac{1}{2}\left(\frac{1}{x+i \epsilon}+\frac{1}{x-i \epsilon}\right)
$$


The first step is to perform the integral

$$
\int d x \frac{1}{2}\left(1-x^{2}\right) \rho_{L}(l x, l) \mathcal{P}\left(\frac{1}{x-x^{\prime}}\right)
$$

via the standard dispersion relation (see [29]) keeping track of the contribution of the large circle at infinity. We obtain

$$
I_{L}^{b}=-\pi \operatorname{Re} \int_{-1}^{1} d x^{\prime} \int_{0}^{\infty} d l\left(1-\frac{l^{2}}{l^{2}-3 m_{g}^{2} Q_{1}\left(x^{\prime}\right)}\right) ; \quad Q_{1}\left(x^{\prime}\right)=\frac{x}{2} \ln \left(\frac{x^{\prime}+1}{x^{\prime}-1}\right)-1,
$$

and performing the integral over $l$ we have,

$$
I_{b}=\frac{\sqrt{3}}{2} \pi m_{g} \int_{-1}^{1} d x^{\prime} \operatorname{Re} \sqrt{Q_{1}\left(x^{\prime}\right)}\left(\ln \left(\sqrt{Q_{1}\left(x^{\prime}\right)}\right)-\ln \left(-\sqrt{Q_{1}\left(x^{\prime}\right)}\right)\right)
$$

Cutting the complex plane along the negative real axis for both the square root function and the logarithm we find,

$$
\left(\ln \left(\sqrt{Q_{1}\left(x^{\prime}\right)}\right)-\ln \left(-\sqrt{Q_{1}\left(x^{\prime}\right)}\right)\right)=-i \pi \operatorname{sgn}\left(x^{\prime}\right)
$$

which gives,

$$
I_{b}=\frac{\sqrt{3}}{2} \pi^{2} m_{g} \operatorname{Im} \int_{-1}^{1} d x^{\prime} \operatorname{sgn}\left(x^{\prime}\right) \sqrt{Q_{1}\left(x^{\prime}\right)}
$$

We do the $x^{\prime}$ integral by writing $f(z)=\sqrt{Q_{1}(z)}$ and choosing the contour shown in Fig. (2). We obtain,

$$
I_{b}=-\pi^{3} m_{g} / 2
$$

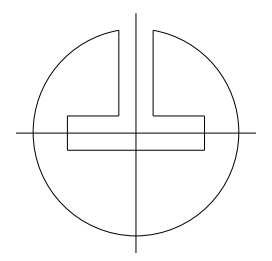

Figure 2: Contour in the complex $x^{\prime}$ plane

Combining (5.5) and (5.11) we have,

$$
I_{L}=\pi^{2} m_{g}\left(\sqrt{3}-\frac{\pi}{2}\right)
$$

\subsection{Transverse Integral}

In this section we consider the transverse integral (5.2). The $x$-integral can be performed in a way similar to the one above, but the result exhibits an ultraviolet divergence in the $l$-integral. To understand this result, we separate the gluon spectral function into the pole piece and the cut piece (see [29]).

The pole piece carries the ultraviolet divergence. This divergence is not physical: it is a consequence of performing an infrared expansion on an integral that is dominated by the ultraviolet part of the phase space (the expansion in $l / q_{0}$ used above is not valid in this case). But the pole part is just the contribution calculated in [9] (up to higher order terms since the transverse gluon has a small thermal mass), the result of which is given in eqs. (1.3), (1.4). 
The cut part of the spectral function does not produce a divergence. We have,

$$
I_{T}^{\mathrm{cut}}=\int_{-1}^{1} \frac{d x}{x} \int_{0}^{\infty} d l l^{2} \beta_{T}(x, l)\left[x^{2}+\left(1-x^{2}\right) \frac{x}{2} \ln \left|\frac{x+1}{x-1}\right|\right]
$$

where

$$
\beta_{T}(x, l)=\frac{-2 \operatorname{Im} \Pi_{T}}{\left(l^{2}\left(x^{2}-1\right)-\operatorname{Re} \Pi_{T}\right)^{2}+\left(\operatorname{Im} \Pi_{T}\right)^{2}}
$$

and $\Pi_{T}$ is given in (2.7a). Since the integration range does not extend from positive to negative infinity we cannot use a sum-rule-like trick to perform the integral. We proceed as follows. The $\int d l$ integral can be done by hand. The only mass scale in the integrand is $m_{g}$ and a simple dimensional analysis shows that the $\int d x$ must be of order $m_{g} \sim g T$. The remaining integral is done numerically to obtain,

$$
I_{T}^{\text {cut }}=-6.67 m_{g}
$$

which is of the same order as the longitudinal contribution.

\section{Conclusions}

In this note we have computed the lepton pair production rate by a static heavy photon at two-loop order (Fig. 11) and focused on the infrared region for the exchanged gluon. The result is free of infrared and collinear divergences, in agreement with a previous study in the case where the external particle was a scalar 22]. As in 22], we find the contribution of the longitudinal gluon to be of order $e^{2} g^{3}$. However, our results differ for the transverse sector. The authors of 22 find that the contribution of the transverse gluon is of order $e^{2} g^{4}$. Our results are as follows:

[1] The infrared approximation used in this computation is valid for the "cut" part $\left(x^{2}<1\right)$ of the gluon spectral function. The contribution for "cut" part is of order $e^{2} g^{3}$.

[2] The infrared region of the gluon phase space does not give the dominant contribution to the pole piece of the gluon spectral function. The pole piece is dominated by large momenta. This means that thermal effects can be neglected and we can use the result obtained with a bare gluon propagator which was calculated previously [9].

The physical picture of the calculated contributions is the following. The one-loop contribution is the annihilation of a quark-antiquark pair, each parton carrying half of the energy of the static photon, namely $q_{0} / 2$. The dominant two-loop contribution describes the scattering of one of the annihilating quark in the medium with an exchange of small momentum of order $l \sim m_{g} \sim g T$. Interestingly, these processes are similar to those which dominate the production of the small mass, high momentum (of $\mathcal{O}(T)$ or higher) lepton pairs, i.e. the other extreme kinematical domain 30. In the static case, higher order loop diagrams may modify the result, as was found to be the case for energetic photons with a small or vanishing invariant mass 31, 32.

We summarize below the result for $\operatorname{Im}_{R}^{\mu}{ }_{\mu}^{\mu}$ up to order $g^{3}$ in perturbation theory :

$$
\operatorname{Im}_{R}^{\mu}=\alpha N_{c} q_{0}^{2}\left[1-2 n_{F}\right]\left[1+C_{F} \frac{g^{2}}{4 \pi^{2}}\left(\frac{3}{4}+1.62 n_{F}\left(1-n_{F}\right) \frac{m_{g}}{T}\right)\right]
$$

The complete result (6.1) shows that the processes considered in this paper can be relatively large when $q_{0} / T$ is not too large. This suggests that these processes may be relevant for lepton pair production from static heavy photons at temperatures where we expect a quark-gluon plasma to be formed $33,34,35$.

\section{Appendix}

As a check of the above results we have derived the transverse gluon contribution using an alternative method. The $\int d p_{0}$ integration is done using Cauchy's theorem in the complex $p_{0}$ plane. The $\int d y$ integration is 
performed second, and then the imaginary part is taken. The $\int d p$ integration is done last. In addition, vertex and self-energy matrix elements are combined before carrying out the integral so that a partial cancellation of terms occurs before integration. We start from (3.2) and (3.6). In the $p_{0}$ complex plane, the integrand has poles above and below the real axis from the propagators, as well as poles on the imaginary axis from the statistical factors $n_{F}\left(r_{0}\right)$ and $n_{F}\left(p_{0}\right)$. First we note that when we close the contour in the upper-half or the lower-half $p_{0}$ plane, the contribution from the poles on the imaginary axis drop out because the difference between the retarded and the advanced propagators vanishes. Second, we use the fact that terms containing only retarded or advanced propagators do not contribute. The product of propagators can thus be simplified so that we obtain:

$$
\begin{aligned}
& \operatorname{Im} \Pi_{R}^{\text {self(b) }{ }_{\mu}^{\mu}}=N_{c} C_{F} e^{2} g^{2} \operatorname{Re} \int \frac{d^{4} P}{(2 \pi)^{4}}\left[n_{F}\left(r_{0}\right)-n_{F}\left(p_{0}\right)\right] \int \frac{d^{4} L}{(2 \pi)^{4}} \operatorname{Tr}_{\rho \sigma}^{(s e l f(b))} n_{B}\left(l_{0}\right) \mathcal{R}^{\rho \sigma} \\
& \times\left[D_{A}(P)\left(D_{R}(R)\right)^{2} D_{R}(R+L)\right] \\
& \operatorname{Im}_{R}^{(\text {vertex })_{\mu}^{\mu}}{ }_{\mu}=N_{c} C_{F} e^{2} g^{2} \operatorname{Re} \int \frac{d^{4} P}{(2 \pi)^{4}}\left[n_{F}\left(r_{0}\right)-n_{F}\left(p_{0}\right)\right] \int \frac{d^{4} L}{(2 \pi)^{4}} \operatorname{Tr}_{\rho \sigma}^{(v e r t e x)} n_{B}\left(l_{0}\right) \mathcal{R}^{\rho \sigma} \\
& \times\left[D_{R}(P) D_{R}(P+L) D_{A}(R) D_{A}(R+L)\right]
\end{aligned}
$$

In the expressions above it is understood that the poles in the statistical weights should be ignored when doing the $p_{0}$ integration in the complex plane. Only the poles from the propagators contribute.

We can now add vertex and self-energy contributions so that the full expresion of the trace on the quark loop multiplied by the appropriate propagators is:

$$
\begin{aligned}
\operatorname{Tr}_{\rho \sigma} & \cdot \text { propagators }= \\
& -4\left[\frac{2 L^{2}\left(R^{\rho} R^{\sigma}+P^{\rho} P^{\sigma}\right)-4 Q^{2} R^{\rho} P^{\sigma}+g^{\rho \sigma} L^{2}\left(Q^{2}+L^{2}-P^{2}-R^{2}-(P+L)^{2}-(R+L)^{2}\right)}{P_{R}^{2} R_{A}^{2}(P+L)_{R}^{2}(R+L)_{A}^{2}}\right. \\
& +Q^{2}\left(\frac{-4 P^{\rho} P^{\sigma}+g^{\rho \sigma}\left((P+L)^{2}-L^{2}\right)}{\left(P_{R}^{2}\right)^{2} R_{A}^{2}(P+L)_{R}^{2}}+\frac{-4 R^{\rho} R^{\sigma}+g^{\rho \sigma}\left((R+L)^{2}-L^{2}\right)}{P_{R}^{2}\left(R_{A}^{2}\right)^{2}(R+L)_{A}^{2}}\right) \\
& \left.+g^{\rho \sigma}\left(\frac{1}{P_{R}^{2}}-\frac{1}{(P+L)_{R}^{2}}\right)\left(\frac{1}{R_{A}^{2}}-\frac{1}{(R+L)_{A}^{2}}\right)+g^{\rho \sigma}\left(\frac{2 Q L}{P_{R}^{2} R_{A}^{2}(P+L)_{R}^{2}}-\frac{2 Q L}{P_{R}^{2} R_{A}^{2}(R+L)_{A}^{2}}\right)\right]
\end{aligned}
$$

where we have used the notation $P_{R}^{2}=\left(p_{0}+i \epsilon\right)^{2}-p^{2}$, etc. As discussed in 32] (see Eq.(3.12) of this reference), this expression already exhibits some cancellations due to gauge invariance. Further simplifications are possible in our case since we are working in the limit of large $q_{0}^{2}=Q^{2}$ and small $\left\{l_{0}^{2}, l^{2}, L^{2}\right\}$. At this point we consider only transverse gluons (longitudinal gluons can be treated using the same method) and identify the terms that contribute to (7.3) to leading order. It has been checked explicitly that all other terms are subdominant. We need to calculate:

$$
\begin{aligned}
& T_{1}=e^{2} g^{2} N_{c} C_{F} \operatorname{Re} \int \frac{d^{4} P}{(2 \pi)^{4}}\left[n_{F}\left(r_{0}\right)-n_{F}\left(p_{0}\right)\right] \int \frac{d^{4} L}{(2 \pi)^{4}} n_{B}\left(l_{0}\right) \rho_{T}(L) \frac{16 Q^{2} R^{\rho} P^{\sigma} P_{T}^{\rho \sigma}(L)}{P_{R}^{2} R_{A}^{2}(P+L)_{R}^{2}(R+L)_{A}^{2}} \\
& T_{2}=e^{2} g^{2} N_{c} C_{F} \operatorname{Re} \int \frac{d^{4} P}{(2 \pi)^{4}}\left[n_{F}\left(r_{0}\right)-n_{F}\left(p_{0}\right)\right] \int \frac{d^{4} L}{(2 \pi)^{4}} n_{B}\left(l_{0}\right) \rho_{T}(L) \frac{16 Q^{2} R^{\rho} R^{\sigma} P_{T}^{\rho \sigma}(L)}{P_{R}^{2}\left(R_{A}^{2}\right)^{2}(R+L)_{A}^{2}}
\end{aligned}
$$

and an equation similar to $T_{2}$ with $P$ and $R$ interchanged. We discuss below the strategy for calculating the necessary integrals. Consider, for example, a term of the form:

$$
\mathrm{Int}=\operatorname{Re} \int p^{2} d p d y d p_{0} \frac{1}{P_{R}^{2}(P+L)_{R}^{2} R_{A}^{2}(R+L)_{A}^{2}}
$$

where the integrals over $l_{0}$ and $l$ have been supressed (see (3.1)). These integrals must be calculated following the method of Sec. 5 and are not discussed in this appendix. We close the $p_{0}$ contour in the lower half-plane. 
There are contributions from the poles of the factors $P_{R}^{2}$ and $(P+L)_{R}^{2}$. In the small $\left\{l_{0}, l\right\}$ limit the contributions from the poles of the factor $(P+L)_{R}^{2}$ will have the same form as the contribution from the poles of $P_{R}^{2}$. Thus we will consider only the poles $p_{0}= \pm p-i \epsilon$ from the factor $P_{R}^{2}$, and multiply the result by a factor of two. This procedure gives rise to an integral of the form,

$$
\begin{aligned}
\operatorname{Re} \int d p_{0} \frac{F\left(p_{0}\right)}{P_{R}^{2}} & =\sum_{n= \pm} \operatorname{Re}\left(\frac{i \pi}{p} F(n p-i \epsilon)\right) \\
& =-\frac{\pi}{p} \operatorname{Im} F(-p-i \epsilon),
\end{aligned}
$$

where we have neglected, in the last line, the pole which will not lead to an imaginary part in the function $F$. Using (7.6) we obtain,

$$
\frac{1}{R_{A}^{2}}=\frac{1}{q_{0}\left(q_{0}-2 p-i \epsilon\right)}, \quad \frac{1}{(P+L)_{R}^{2}}=\frac{1}{c-b y}, \quad \frac{1}{(R+L)_{A}^{2}}=\frac{1}{a-b y}
$$

where

$$
\begin{aligned}
a-b & =\left(q_{0}+l_{0}+l\right)\left(q_{0}-2 p+l_{0}-l\right)-i \epsilon, & & a+b=\left(q_{0}+l_{0}-l\right)\left(q_{0}-2 p+l_{0}+l\right)-i \epsilon \\
c-b & =\left(l_{0}+l\right)\left(-2 p+l_{0}-l\right), & c+b & =\left(l_{0}-l\right)\left(-2 p+l_{0}+l\right) .
\end{aligned}
$$

We note that when the retarded propagator $1 /(P+L)_{R}^{2}$ is evaluated at the pole of the retarded function $1 / P_{R}^{2}$, the $i \epsilon$ piece cancels exactly and thus the variables $b-c$ and $b+c$ in (7.8) do not contain an $i \epsilon$ prescription. Carrying out the angular integration on $y$, one obtains:

$$
\int_{-1}^{1} d y \frac{1}{(a-b y)} \frac{1}{(c-b y)}=\frac{1}{2 q_{0} p l} \frac{1}{\left(q_{0}-2 p+2 l_{0}-i \epsilon\right)}\left(\ln \left(\frac{a-b}{a+b}\right)-\ln \left(\frac{c-b}{c+b}\right)\right) .
$$

Combining (7.5), (7.6), (7.7) and (7.9) we have,

Int

$$
=-2 \pi \int d p p \operatorname{Im}\left[\frac{1}{2 q_{0} p l} \frac{1}{q_{0}\left(q_{0}-2 p-i \epsilon\right)} \frac{1}{\left(q_{0}-2 p+2 l_{0}-i \epsilon\right)}\left(\ln \left(\frac{a-b}{a+b}\right)-\ln \left(\frac{c-b}{c+b}\right)\right)\right] .
$$

where

$$
\begin{aligned}
\frac{1}{\left(q_{0}-2 p-i \epsilon\right)\left(q_{0}-2 p+2 l_{0}-i \epsilon\right)}= & \frac{1}{2 l_{0}}\left(\mathcal{P}\left(\frac{1}{q_{0}-2 p}\right)-\mathcal{P}\left(\frac{1}{q_{0}-2 p+2 l_{0}}\right)\right) \\
& +\frac{i \pi}{2 l_{0}}\left(\delta\left(q_{0}-2 p\right)-\delta\left(q_{0}-2 p+2 l_{0}\right)\right) .
\end{aligned}
$$

There is also an imaginary piece to the first logarithm in (17.10). We use the relation $\ln (x-i \epsilon)=\ln (|x|)-$ $i \pi \theta(-x)$ to separate real and imaginary pieces and obtain,

$$
\int_{0}^{\infty} d p \ln \left(\frac{a-b}{a+b}\right)=\int_{0}^{\infty} d p\left(\ln \left(\frac{q_{0}+l_{0}+l}{q_{0}+l_{0}-l}\right)+\ln \left(\frac{q_{0}-2 p+l_{0}-l}{q_{0}-2 p+l_{0}+l}\right)\right)-i \pi \int_{\left(q_{0}+l_{0}-l / 2\right.}^{\left(q_{0}+l_{0}+l\right) / 2} d p
$$

To complete the evaluation of (7.10) we take the product of (7.11) and (7.12) and extract the imaginary part. There are two contributions: one contains the imaginary part of (7.11) which contains a delta function that allows us to do the $\int p$ integral, and the second contains the imaginary part of (7.12). This factor contains an integral in $p$ phase-space that is restricted to a small interval around $q_{0} / 2$ which can be calculated by expanding around the value $p=q_{0} / 2$. This procedure leads to the appearance of a factor $n_{F}^{\prime \prime}$ as defined in (3.15). The $\int d p$ integration can then easily be done. The calculation of terms like $T_{2}$ is carried out in the same way, the double pole being handled via an integration by parts on the momentum variable after taking the residue of the single pole via the contour integration. Finally we obtain:

$$
T_{1}=\frac{e^{2} g^{2}}{(2 \pi)^{4}} N_{c} C_{F} \frac{q_{0}^{2}}{T} n_{F}^{\prime \prime} \int \frac{d x}{x} \int d l l^{2} \rho_{T}(x, l)\left[x^{2}-\frac{1}{3}+\left(1-x^{2}\right) \frac{x}{2} \ln \left(\frac{1+x}{1-x}\right)\right]
$$




$$
T_{2}=\frac{e^{2} g^{2}}{(2 \pi)^{4}} N_{c} C_{F} \frac{q_{0}^{2}}{T} n_{F}^{\prime \prime} \int \frac{d x}{x} \int d l l^{2} \rho_{T}(x, l) \frac{1}{6} .
$$

Adding (7.13) and twice (7.14) gives the full transverse contribution as calculated in (5.2).

\section{References}

[1] T. Peitzmann and M. H. Thoma, Phys. Rept. 364 (2002) 175

[2] M. M. Aggarwal et al. [WA98 Collaboration], Phys. Rev. Lett. 85 (2000) 3595

[3] M. M. Aggarwal et al. [WA98 Collaboration], arXiv:nucl-ex/0006007

[4] D. K. Srivastava, Eur. Phys. J. C 10 (1999) 487 [Erratum-ibid. C 20 (2001) 399].

[5] D. K. Srivastava and B. Sinha, Phys. Rev. C 64 (2001) 034902

[6] P. Huovinen, P. V. Ruuskanen and S. S. Rasanen, Phys. Lett. B 535 (2002) 109

[7] J. Sollfrank, P. Huovinen, M. Kataja, P. V. Ruuskanen, M. Prakash and R. Venugopalan, Phys. Rev. C 55 (1997) 392

[8] L.D. Lc Lerran, T. Toimela, Phys. Rev. D 31 (1985) 545.

[9] T. Altherr and P. Aurenche, Z. Phys. C 45 (1989) 99.

[10] R. D. Pisarski, PhysicaA 158 (1989) 146.

[11] E. Braaten and R. D. Pisarski, Phys. Rev. Lett. 64 (1990) 1338; Nucl. Phys. B 337 (1990) 569; Nucl. Phys. B 339 (1990) 310.

[12] J. Frenkel and J. C. Taylor, Nucl. Phys. B 334 (1990) 199; Nucl. Phys. B 374 (1992) 156.

[13] T. Kinoshita, J. Math. Phys. 3 (1962) 650.

[14] T. D. Lee and M. Nauenberg, Phys. Rev. 133 (1964) B1549.

[15] R. Baier, B. Pire and D. Schiff, Phys. Rev. D 38 (1988) 2814.

[16] T. Altherr, P. Aurenche and T. Becherrawy, Nucl. Phys. B 315 (1989) 436.

[17] T. Altherr and T. Becherrawy, Nucl. Phys. B 330 (1990) 174.

[18] Y. Gabellini, T. Grandou and D. Poizat, Annals Phys. 202 (1990) 436.

[19] A. Majumder and C. Gale Phys. Rev. C 65 (2002) 055203.

[20] J. Cleymans and I. Dadic, Z. Phys. C 42 (1989) 133.

[21] S.M.H. Wong, Z. Phys. C 58 (1993) 159.

[22] M. Le Bellac and P. Reynaud, Nucl. Phys. B 416 (1994) 801.

[23] N. P. Landsman and C. G. van Weert, Phys. Rept. 145 (1987) 141.

[24] H. A. Weldon, Phys. Rev. D 26 (1982) 1394.

[25] V. V. Klimov, Sov. Phys. JETP 55 (1982) 199 [Zh. Eksp. Teor. Fiz. 82 (1982) 336].

[26] P. Aurenche and T. Becherrawy, Nucl. Phys. B 379, 259 (1992).

[27] P. Aurenche, T. Becherrawy and E. Petitgirard, arXiv:hep-ph/9403320. 
[28] P. Aurenche, F. Gelis, R. Kobes and E. Petitgirard, Z. Phys. C 75 (1997) 315

[29] M. LeBellac, "Thermal Field Theory," Cambridge University Press, Cambridge, United Kingdom, 1996.

[30] P. Aurenche, F. Gelis and H. Zaraket, JHEP 0207 (2002) 063.

[31] P. Arnold, G.D. Moore and L.G. Yaffe, JHEP 0111 (2001) 057; JHEP 0112 (2001) 009; JHEP 0206 (2002) 030.

[32] P. Aurenche, F. Gelis, G.D. Moore and H. Zaraket, JHEP 0212 (2002) 006.

[33] T. Ullrich et al. [CERES Collaboration], Nucl. Phys. A 610 (1996) 317C.

[34] B. Lenkeit et al. [CERES-Collaboration], Nucl. Phys. A 661 (1999) 23

[35] D. Adamova et al. [CERES/NA45 Collaboration], 